Document downloaded from:

http://hdl.handle.net/10251/43823

This paper must be cited as:

Hernández, L.; Baladrón Zorita, C.; Aguiar Pérez, JM.; Carro, B.; Sanchez-Esguevillas, A.; Lloret, J.; Chinarro, D.... (2013). A Multi-Agent System Architecture for Smart Grid Management and Forecasting of Energy Demand in Virtual Power Plants. IEEE Communications Magazine. 51(1):106-113. doi:10.1109/MCOM.2013.6400446.

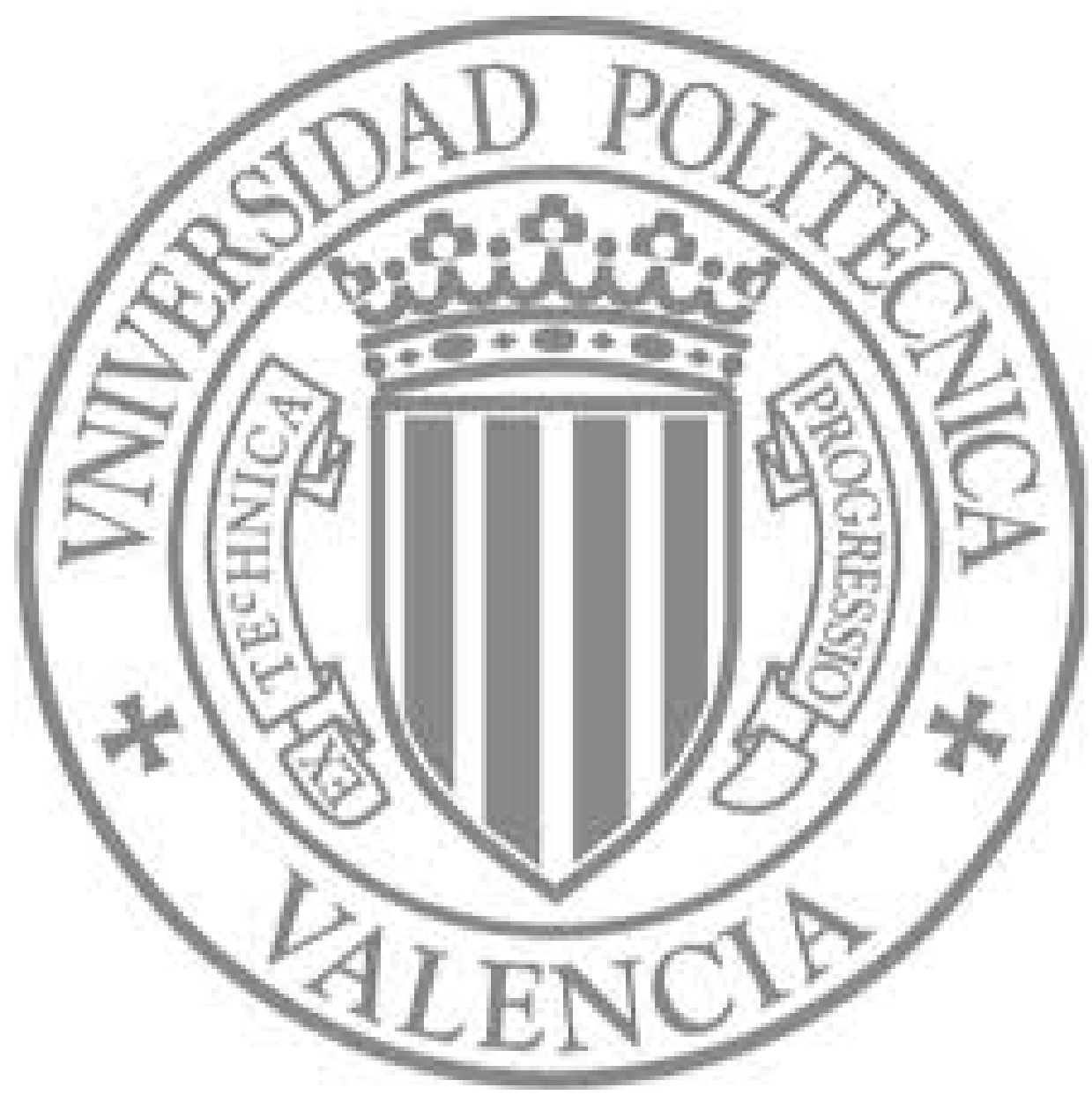

The final publication is available at

http://dx.doi.org/10.1109/MCOM.2013.6400446

Copyright Institute of Electrical and Electronics Engineers (IEEE) 


\title{
A Multi-Agent-System Architecture for Smart Grid Management and Forecasting of Energy Demand in Virtual Power Plants
}

\author{
Luis Hernández ${ }^{1}$, Carlos Baladrón ${ }^{2}$, Javier M. Aguiar ${ }^{2}$, Belén Carro², Antonio \\ Sánchez-Esguevillas ${ }^{2}$, Jaime Lloret ${ }^{3}$, David Chinarro ${ }^{4}$, Jorge J. Gómez ${ }^{5}$, Diane \\ Cook $^{6}$ \\ ${ }^{1}$ CIEMAT (Centro de Investigaciones Energéticas, Medioambientales y \\ Tecnológicas), ${ }^{2}$ Universidad de Valladolid, ${ }^{3}$ Universidad Politécnica de Valencia, \\ ${ }^{4}$ Universidad de San Jorge, ${ }^{5}$ Universidad Complutense de Madrid, ${ }^{6}$ Washington \\ State University
}

\begin{abstract}
Recent technological advances in the Power Generation and Information Technologies areas are helping to change the modern electricity supply system, in order to comply with higher energy efficiency and sustainability standards. Smart Grids are an emerging trend which introduces intelligence in the power grid to optimize resource usage. In order for this intelligence to be effective, it is necessary to retrieve enough information about the grid operation together with other context data such as environmental variables and intelligently modify the behaviour of the network elements accordingly. This paper presents a Multi-Agent System model for Virtual Power Plants, a new power plant concept in which generation no longer occurs in big installations, but is the result of the cooperation of smaller and more intelligent elements. The proposed model is not only focused on the management of the different elements, but includes a set of agents which are embedded with Artificial Neural Networks for collaborative forecasting of disaggregated energy demand of domestic end users, the results of which are also shown in this paper.
\end{abstract}

Keywords: Distributed Intelligence; Multi-Agent System; Smart Grid; Virtual Power Plant.

\section{Introduction}

Following the principles of "green" trends and the restrictions imposed by the ever increasing demand and prices of oil and other fuels, Power Generation and Distribution systems have been constantly evolving during the last years into more sustainable models compatible with environmental protection. This will be the key to achieve a sustainable (energy) economy in accordance with the provisions of the Treaty of Lisbon.

In addition, energy markets are facing a set of global challenges. These include: 
- Global deregulation.

- Innovative elements are being gradually integrated into the Energy Grid, such as Distributed Energy Resources (DER) or Distributed Generation (DG) [1]: compact, sometimes mobile, energy generation devices (diesel engines, fuel cells, thin film photovoltaics, and mini wind turbines).

- The imminent massive irruption of electric vehicles.

- Increasing consumer involvement: consumers modify their behaviour according to green trends and financial savings [2].

Moreover, utilities have been encouraged through government policies to incorporate new smart devices. For example, Smart Meters are deployed at users' homes and data collectors are installed in electric substations. Additionally, Smart Homes allow autonomous control of electric devices [3, 4].

This increasing intelligence of power grid nodes has led to the concept of Smart Grids: intelligent power grids capable of performing autonomous adaptation of its elements in order to optimize resource consumption (e.g., minimize energy transport due to localized production, reshape demand through the usage of automated devices in smart homes).

Inside Smart Grids, a new energy production model called a Virtual Power Plant (VPP) [5] emerges, in which the power plant is no longer a monolithic installation, but an aggregation of smaller cooperating intelligent elements. The aggregation of generators and loads running together covers a physical or logical autonomous unit where all elements operate coherently, as in an industrial park, a residential area with solar panels or the distributed resources of a utility.

However, management of these VPPs poses a challenge for Information Technologies. VPPs comprise a plethora of different elements designed to solve local problems, but that need to interact together in order to behave as a unit (the VPP). Protocols and tools are needed to let these elements communicate, discriminate and decide among the different (and sometimes diverging) requirements, make decisions affecting several entities, and coordinate coherent behaviours to carry out complex tasks. At the same time, the VPP devices are extremely heterogeneous: they are built and managed by very different organizations with very different purposes, and follow different standards for control and communication. 
In addition to big plants, the power grid landscape of the future embraces many smaller elements: solar panels in houses, small wind turbines for residential areas, and small plants in industrial parks. While these elements can operate isolated, their integration will facilitate a more efficient usage of the entire energy resource base. It is possible to imagine a VPP autonomously redirecting energy from solar panels of empty houses to cover peak demands from other homes (billing the users accordingly), automatically selecting the best moment to recharge electric vehicles (taking advantage of wind peaks during the night), or sell surplus energy to a nearby industrial park.

In the current state of the art, Multi-Agent System (MAS) [6] models have been proposed to control elements of Smart Grids, most notably for large area power networks (such as supporting an intelligent energy market) [7, 8]. MAS are a novel computing paradigm in which multiple entities within an environment influence and are influenced by other entities in the same environment. Additionally, load forecasting is a recurrent problem in energy grids, and as such many solutions have been proposed [9], although most are designed for large geographic areas.

In this paper, we introduce an architectural solution to the challenge of VPP management through the application of a MAS and propose a solution for energy load forecasting in small (microgrid) scale environments, which will be employed by all its elements to adapt their behaviour in advance, facilitating demand reshaping and generation planning. While existing works [7-9] focus on large area management and large area load forecasting, our model manages VPPs and load forecasting for much smaller and more detailed environments.

This paper is organized as follows: Section 2 defines in detail the concept of VPP. Section 3 describes the new possibilities offered by ITs. Section 4 presents the proposed architectural model relying on a Multi-Agent System and the demand forecasting system validated with real data. Section 5 ends the paper with the conclusions.

\section{Virtual Power Plants}

As new intelligent agents and Distributed Energy Resources (DERs) are incorporated into power generation, transforming old infrastructures into Smart 
Grids, a new paradigm needs to be developed to ensure coordination among all the implied entities. VPPs emerge as a decentralized, self-organized intelligent solution.

A single small generation unit cannot offer cost-effective capacity, reliability, flexibility and controllability in an electricity market. A VPP is a cluster of distributed generation installations that operate independently, but together could be seen as a single generation plant with its own generation schedule and limits, as well as its own operating cost and demand characteristics. A VPP plays two main roles. The first one is Commercial Virtual Power Plant (CVPP). It is aimed at the economic optimisation. CVPP contracts power from DERs in exchange for optimised generator revenues, compiling their technical and economical parameters and building an optimized bid/offer table. Once bids are accepted, CVPP controls contract execution. The second one is Technical Virtual Power Plant (TVPP). It manages optimal and secure operation of the system according to physical constraints and ensures the technical feasibility of the generation/consumption program based on date submitted by the CVPP.

In short, VPPs facilitate the integration of new smart devices and DERs into the electricity wholesale market, offering system management and support services. Figure 1 shows the elements comprising a VPP, such as generators (PV plants, wind plants, biomass plants), consumers (smart homes and smart buildings) and some producers/consumers (electric vehicle and storage).

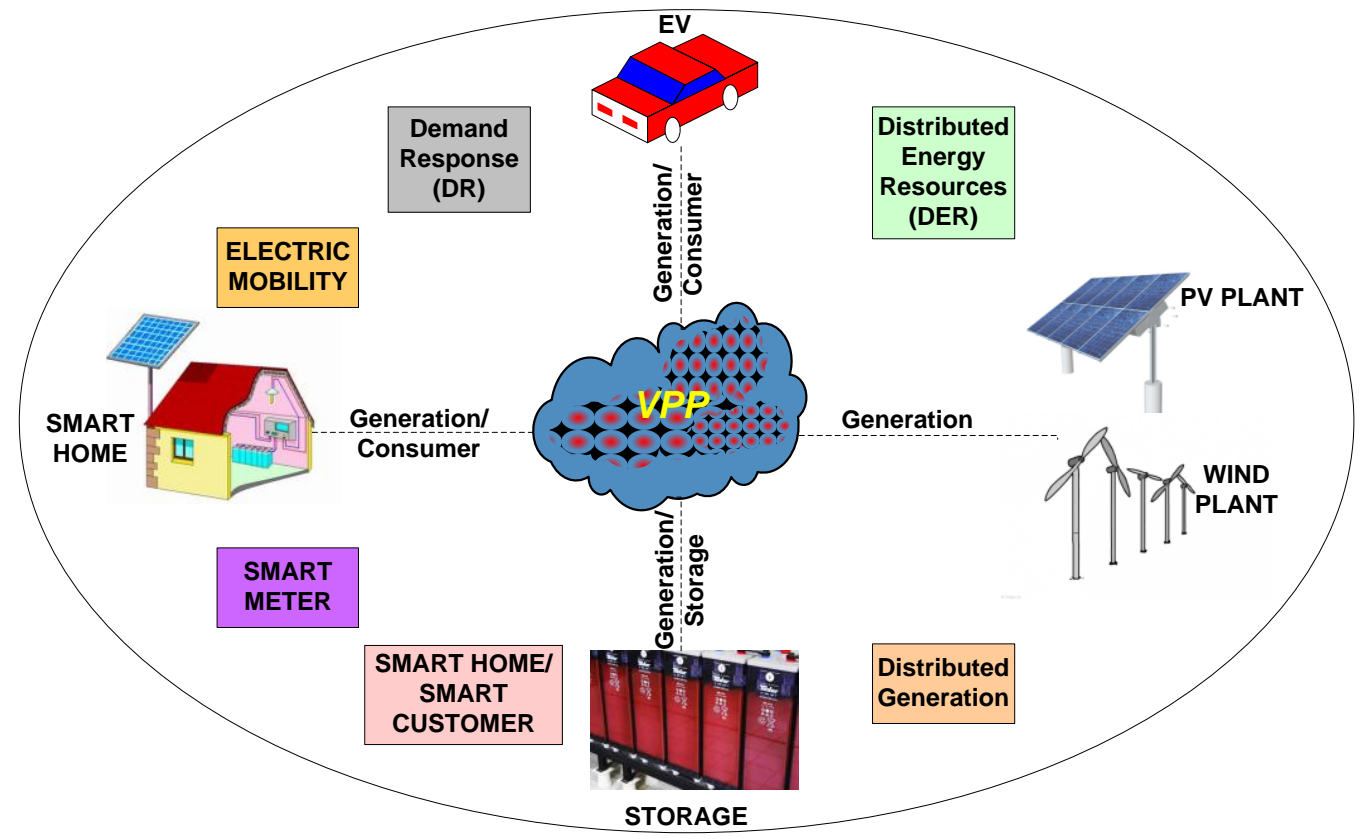


Figure 1. Elements of a Virtual Power Plant.

Moreover, VPPs treat information and actors separately from the physical components. For example, generators in separate locations can be controlled coherently to optimize their performance both locally in each of their physical environments and globally, as a single entity connected to the power grid; similarly, a physical area can house several VPPs simultaneously.

Finally, VPPs utilize security measures to guarantee data integrity and privacy. This has become a critical research field $[10,11]$ because VPPs handle privacy and personal information. For example, smart meters disaggregate demand and consumption patterns while smart homes and next generation electric vehicles monitor the behaviour of their users. These VPPs are subject to failures and may be the object hostile attacks and viruses.

\section{Information Technologies in the Smart Grid scenario}

\subsection{Information Technologies in VPP Physical Structure}

VPPs rely on Distributed Intelligence (DI) that monitors and controls all their elements. Figure 2 shows the generic control architecture of a VPP. The VPP data flow begins with data sources such as smart meters (SMs) or weather stations and sends data to the intelligent agents in low-voltage (LV) substations via data concentrator (DC) systems. Most basic functions are performed at the lowest level of the hierarchical structure, where devices make decisions regarding their own local operations, such as activating a generator or switching off a smart home device. Virtual Power Plant Control (VPPC) coordinates these operations according to the global internal status of the VPP [12], and provides interfaces with the external power grid (represented by the second VPP in Figure 2) according to the external status of other smart elements. For instance, through these VPP interfaces, companies may collectively facilitate the creation of a smart energy market, or VPPs of several industrial parks and residential areas can exchange load and generation information to share energy locally instead of buying it from a global market. 


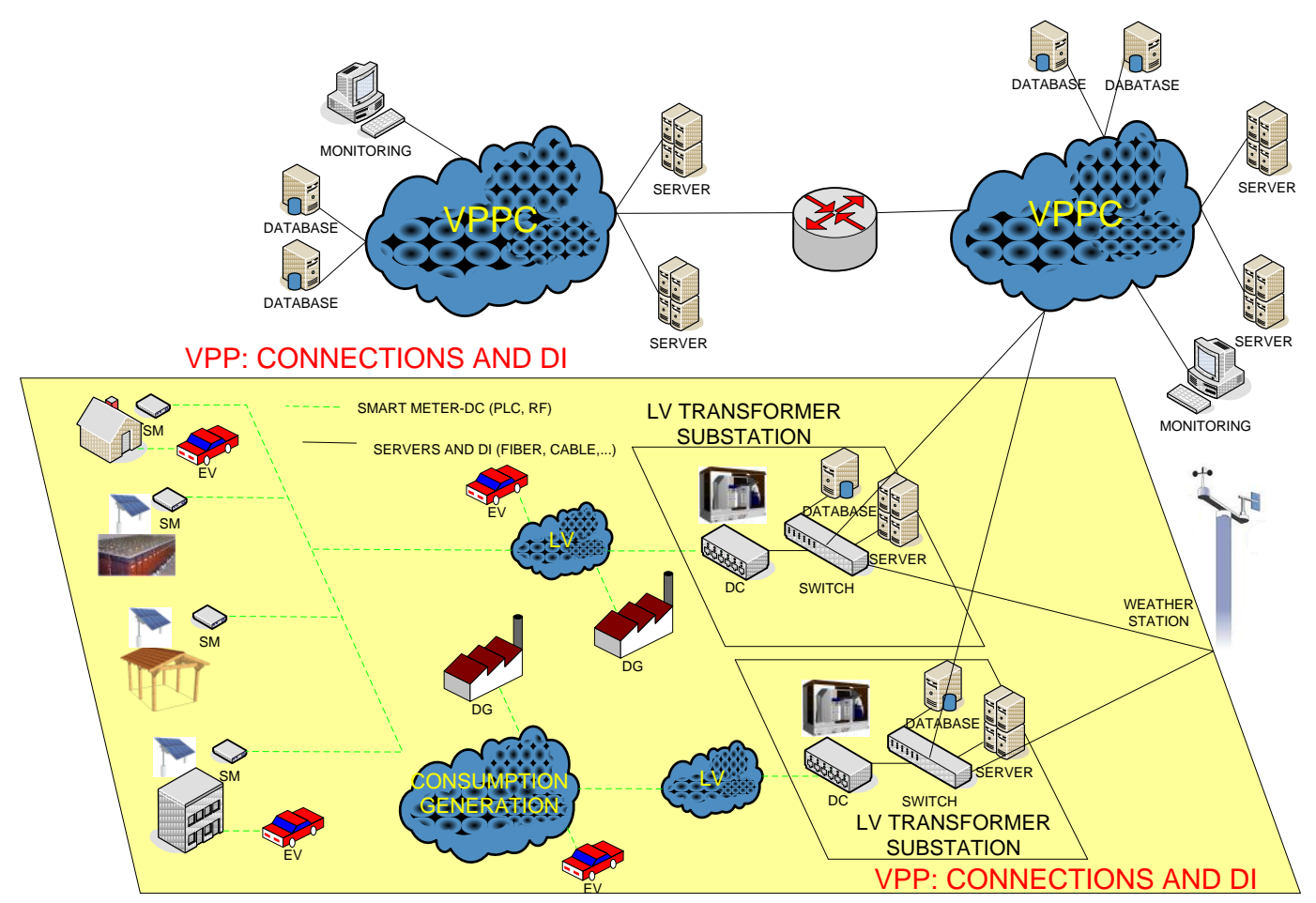

Figure 2. Distributed intelligence via a VPP.

\subsection{Control Processes within a Virtual Power Plant}

Three different types of processes have to be controlled within a VPP: marketrelated issues (Commercial VPP - CVPP), those related to performance and functional problems (Technical VPP - TVPP), and those involving both. Power grid agents and VPP external processes are listed here, together with the corresponding TVPP or/and CVPP:

- State of Network (TVPP): Propagates status (e.g., events, failures)

- State of Generation (CVPP and TVPP): Shares information about current generation capacity and generation forecasts

- State of Demand (CVPP and TVPP): Distributes information about current demand and demand forecasts

- Bids (CVPP and TVPP) : VPP requests/offers energy from/to other external power grid elements

- Tariff (CVPP): Disseminates energy prices offered by the VPP

- Roaming of Generators and Loads (CVPP and TVPP): Shares information about elements switching dynamically between VPPs

Internal VPPC control processes are listed next, with the corresponding TVPP or/and CVPP: 
- Troubleshooting (TVPP): Manages potential network and communication failures

- Generation Control (CVPP and TVPP): Ensures distributed generation elements do not cause grid imbalances

- Generation Forecasting (CVPP): Predicts generation capabilities per element

- Demand Control (CVPP and TVPP): Controls energy demand in its area through customer rates and on the basis of contracts

- Demand Forecasting (CVPP): Predicts end users' demand for energy (decentralized in the case of distributed intelligence near end-use points)

- Energy Balance Management (CVPP): Offers different tariffs to optimize the final balance, in accordance with the current VPP control strategy

- Tariff Updater (CVPP): Informs customers, in real time, about rates set by the Energy Balance Manager

- Customer Control (CVPP and TVPP): Manages customer services

- Network Stability Control (TVPP): Monitors and controls VPP grid performance, quality, reliability and safety parameters

- Communication with other VPPs (CVPP and TVPP): Communicates bidirectionally to other elements

- Weather Station Control (TVPP): Manages meteorological station(s), collects and processes their data, and serves raw and processed information to other architecture elements (e.g., Demand and Generation Forecasting)

- Storage Control (CVPP and TVPP): Monitors and controls VPP energy storage.

\subsection{Multi-Agent System (MAS) Approach}

The VPP environment is a complex structure of decision-making processes running separately but interdependently. Extremely distributed architectures are difficult to control using traditional centralized approaches. Therefore, a new control system needs to be implemented, and the MAS programming paradigm is well suited because, on one hand, in MAS, many actors interact by competing or co-operating. Local software agents focus on the interest of local subsystems, and they influence the global system by negotiating with other software agents, and, in the other hand, MASs implement decision-making processes in an open, flexible and extensible way.

This work presents a MAS model for VPPs in two aspects: demand forecasting and coordination of producers/consumers in order to balance energy production. The first is a pure agent solution, since it does not focus on creating a framework, but on coding the solution following MAS principles. Therefore, the forecasting algorithm is implemented using agents. 
The second aspect is approached differently. Rather than implementing the distributed algorithm, a MAS framework is created implementing a model of the different roles and tasks involved in a VPP, and coding the behaviour of each agent. The design of this framework is accomplished using an Agent Oriented Software Engineering solution named INGENIAS. INGENIAS follows the Model Driven Paradigm, therefore the diagrams we show are actually converted into program code or documents.

\section{Architectural model for management and forecasting}

\subsection{A MAS oriented framework for VPP control}

The MAS is designed taking into account the behaviour we expect from VPP. It is a design inspired by working group discussions during the SOAR workshop [13]. These behaviours are expressed in three use cases where three actors participate. There is a Producer that generates the electricity; Consumers which are regular users demanding a fixed amount of power; and Flexible Consumers that make special deals to reduce power consumption when there is a shortage. The market is assumed to regulate power consumption by means of consumers that agree to consume less power when needed in exchange for specified benefits. The three use cases defined are as follows:

- A producer identifies a power shortage. In this case, the producer detects the problem and contacts all flexible consumers to ask them to reduce their power consumption.

- A consumer identifies a power shortage and contacts the producer. The consumer together with the producer need to investigate what has happened.

- A consumer requires more power. Some installations, like a big factory, may occasionally demand additional power. In this case, the producer must contact other flexible consumers to ask them to reduce power consumption.

We present a MAS that addresses these use cases. It has been designed and implemented using the INGENIAS MAS methodology [14]. System agents are represented through roles which are reusable pieces of functionality. System 
agents are represented in Figure 3.b and they coordinate agents already identified in the case studies: Producer, Consumer, and Flexible Consumer. Each role pursues a goal, expressed with the GTPursues relationship. For example, in addition to consuming power, a Flexible Consumer tries to reduce its consumption when possible or when requested. The roles are expected to fulfil their goals and may extend other roles using the ARoleInheritance relationship, which implies the extending role acquires all goals and associated tasks from the parent role.

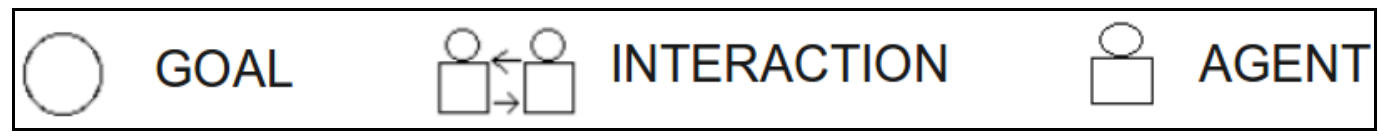

(a)

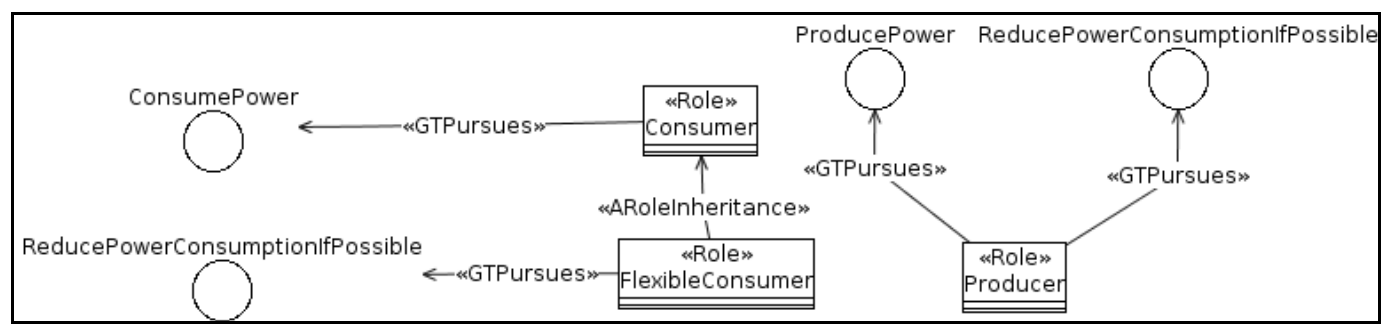

(b)

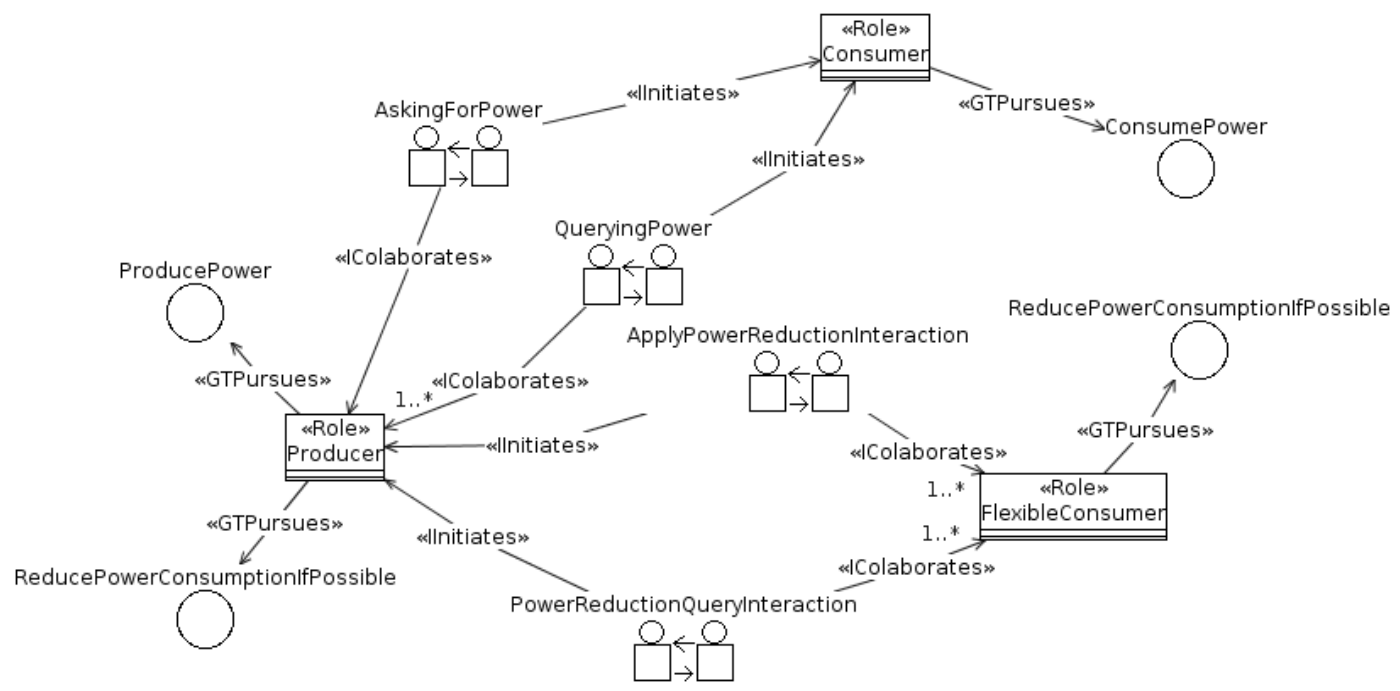

(c)

Figure 3. MAS Model Management and Forecasting System. (a) INGENIAS Notation (b) Actors; (c) Interactions among actors.

Agents are expected to interact in order to realise the use cases previously discussed. The interactions depicted in Figure 3.c embody agent information exchanges. In an interaction there is always one, and only one, agent starting the 
interaction, named the initiator (relationship IInitiates), and at least another agent is waiting to interact, which are called collaborators (relationship IColaborates). Figure 3.c illustrates that a Consumer asks Producers what power can be arranged and at what prices. It is a one-to-many interaction, which means all power grid producers are queried. Once a single producer is selected, the arrangement is accomplished in a different interaction, AskingForPower. These interactions can be started when there is need for additional power (third use case) or there is a power shortage detected by the client (second use case). In the latter case, the client would adhere to new power sources. When there is insufficient energy to satisfy the demand and the producer knows this (first use case), it interacts with clients asking Flexible Consumers to reduce their power consumption. When sufficient power reduction is achieved, the Apply Power Reduction Interaction is started.

We have implemented a fully-operation MAS based on the INGENIAS modeldriven features. Figure 4 shows one of the screens used by the INGENIAS infrastructure to debug the activities performed by the agents. Because it is model driven and there are computational representations for the elements depicted in Figure 3, INGENIAS uses the same elements to communicate with the developer. This way, the developer receives debugging information using the same concepts applied during the design. In this case, the focus of the debugging is a Consumer activity which takes as input a Power Shortage event. This kind of event would be expected from devices detecting energy drops. The designed behavior for the task implies starting an interaction of type Querying Power (see Figure 3.c) with alternative electricity producers. The requested amount of $\mathrm{kW}$ from each one is stored in a slot of the Power Query entity. Without the debugging mode, the agent would just proceed automatically and get the requested amount of electricity. 


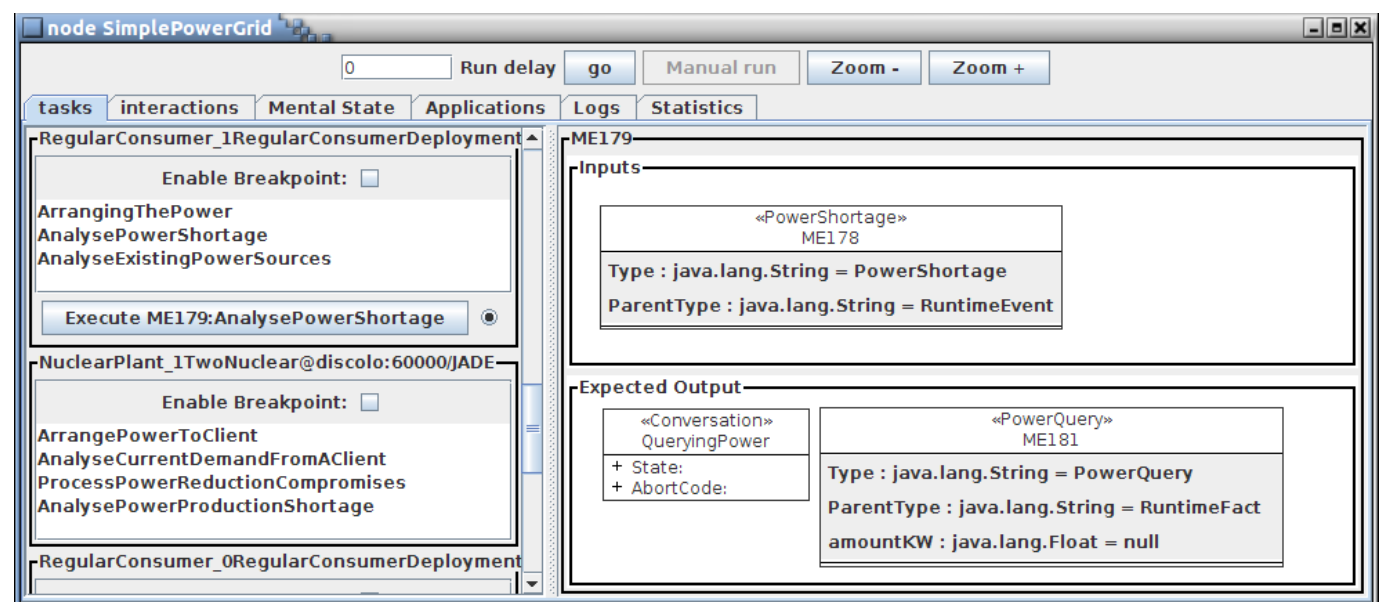

Figure 4. MAS specification execution with INGENIAS.

\subsection{MAS model for Smart Home/Smart House Energy Demand Forecasting}

Of all the control processes explained previously, one of the most critical for the flexible operation of the VPP is Demand Forecasting, which allows the VPP to know in advance the amount power that will be required so that generation planning is feasible. Here we described our MAS implementation of the Demand Forecasting System (DeF) for a VPP.

The objective of the MAS Demand Forecasting system is to forecast VPP users' energy demand by disaggregated sectors. To perform this task, the VPP needs to communicate with other Agents and collaborate with the other processes to ensure VPP stability. DeF agent tasks include:

- DeF - Historical Control Agent (DeF-HcA): This agent decides what historical data will be used in forecast-network retraining.

- DeF - Smart Meter Control Agent (DeF-McA): This agent is responsible for ensuring that smart meters are sending consumption data correctly.

- DeF - Smart Home/House Data Control Agent (DeF-DcA): These agents coordinate with the DeF-McA to verify the relevancy of the obtained data, and report this data to the pre-processing agent.

- DeF - Data Preprocessing Agent (DeF-DpA): These agents are responsible for standardizing the data entered into the database, and for detecting and solving potential irregularities. 
- DeF - Forecasting Agent (DeF-FoA): These agents are in charge of triggering demand forecasting in accordance with a common clock controlled by the DeF system.

- DeF - Retraining Control Agent (DeF-ReA): This agent is in charge of controlling when the forecasting architecturel model needs to be retrained.

- DeF - New Smart Home/House Control Agent (DeF-NcA): These agents are in charge of creating all needed agents and reporting new customer incorporations to the other agents. They also delete agents when a client no longer uses VPP services.

- DeF - External Control Agent (DeF-EcA): The task of these agents is to communicate with the DeF system to learn about customer exchanges between different VPPs and to report the other involved VPP agents.

\subsection{Demand Forecasting}

The system presented in the previous section utilizes a Demand Forecasting subsystem, depicted in Figure 5. This subsystem is based on Artificial Neural Networks (ANN), and, while the literature presents a lot of examples of demand prediction in aggregated environments such as countries, this one is aimed at providing a prediction of the power demand in small environments such as small towns.

ANNs are a computing paradigm based on the aggregation of small computing elements, called neurons, which are interconnected resembling the operation of the human brain. The ANN can present several different architectures, and is normally configured to learn from experience when confronted with a base truth known a priori. The complete forecasting system is shown in Figure 5.a, where load curves and weather variables are introduced into the database (as weather variables have a clear influence on electric demand in a VPP scenario [14]).

The first stage is ANN Self-Organizing Map (SOM) for unattended classification of different training 24-hour demand patterns. Demand patterns with similar features are clustered together. One cluster could represent the smallest consumption days, such as summer weekends and bank holidays, while a different one could represent the highest consumption days, such as weekdays in winter. This stage is represented in Figure 5.b, and uses the following parameters as 
input: three weather variables (average temperature, global solar radiation and relative humidity), type of sector (industrial $=0$, commercial $=1$, domestic $=2$, administration $=3$ and other uses $=4)$, day of the week (Sunday $=0$, Monday $=1, \ldots$, Friday $=5$ and Saturday=6), month (January=1, February $=2, \ldots$, November $=11$ and December $=12$ ), and load curve (24 demand values, one value for each hour of the day).

The second stage is an algorithm to select to which cluster to which a day belongs using weather variables, calendar (day of the week, month and holidays or working days) and load curve (24 values for the load curve of the previous day and the first two hours of demand).

Finally, a third stage is employed in which a set of ANNs, based on a MultiLayer Perceptron (MLP), one for each of the clusters identified, forecast the following 22 hours of demand (ANN output). This is represented in Figure 5.c with the following input variables for each training pattern:

- $\quad L_{d 1}$ and $L_{d 2}$ : represent the 2 values for the load curve of the next day, so that generation/storage planning can be made in advance by the appropriate agents of the system.

- $\quad L_{(d-1) 1}, L_{(d-1) 2}, L_{(d-1) 3}, \ldots, L_{(d-1) 24}$ : represent the 24 values for the load curve of the previous day.

- Day of the week $d-1$ : presented as the two terms sine and cosine (to improve ANN performance), $\sin [(2 \cdot \pi \cdot d a y) / 7](d-1)$ and $\cos [(2 \cdot \pi \cdot d a y) / 7](d-$ 1), with days ranging from 0 to 6 (Sunday=0, Monday $=1, \ldots$, Friday=5, Saturday=6).

- Month $d-1$ : presented as two terms sine and cosine, $\sin [(2 \cdot \pi \cdot d a y) / 12](d-1)$ and $\cos [(2 \cdot \pi \cdot d a y) / 12](d-1)$, with months ranging from 1 to 12 $($ January $=1$, February=2,.., November $=11$, December $=12)$.

As shown in Figure 5.c, the three stages are connected, although the first stage is performed off-line. 

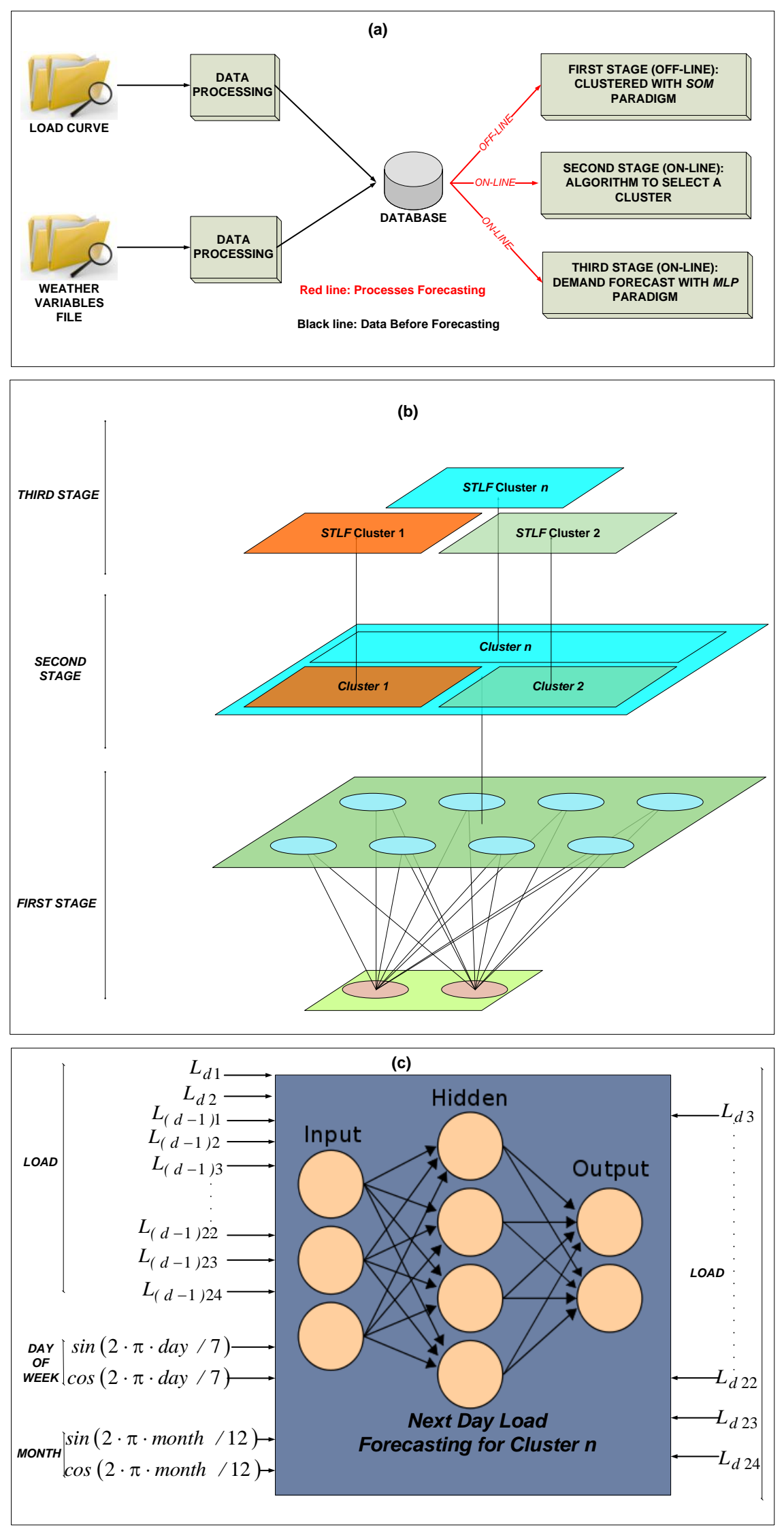

Figure 5. (a) System Complete; (b) representation of the three stages; c) inputs and outputs for $M L P$ models. 
The demand forecasting sub-system is validated with hourly electric demand data from Soria, a small size town in Spain, from the $1^{\text {st }}$ of January, 2008 to the $31^{\text {st }}$ of December, 2010, obtaining a mean prediction error as low as $1.5 \%$, depending on the cluster considered.

Figure 6 illustrates an example of the ANN model. Figure 6.a presents the clustered load curves after the first stage, with each cluster identified by a different colour. It is easy to see how daily load patterns with similar features are grouped together. There are several facts worth mentioning about the load: a region of low energy consumption is observed during the night hours, between $22 \mathrm{~h}$ and $8 \mathrm{~h}$, while two peaks are found normally at $11 \mathrm{~h}-12 \mathrm{~h}$ and $18 \mathrm{~h}-19 \mathrm{~h}$; during the low consumption period, patterns are quite similar, and differences increase during the high one.

Figure 6.b presents an example load curve forecasting for one day, with the target load curve in black, the prediction in red, and the other members of the cluster to which that specific input was assigned in green. 
(a)

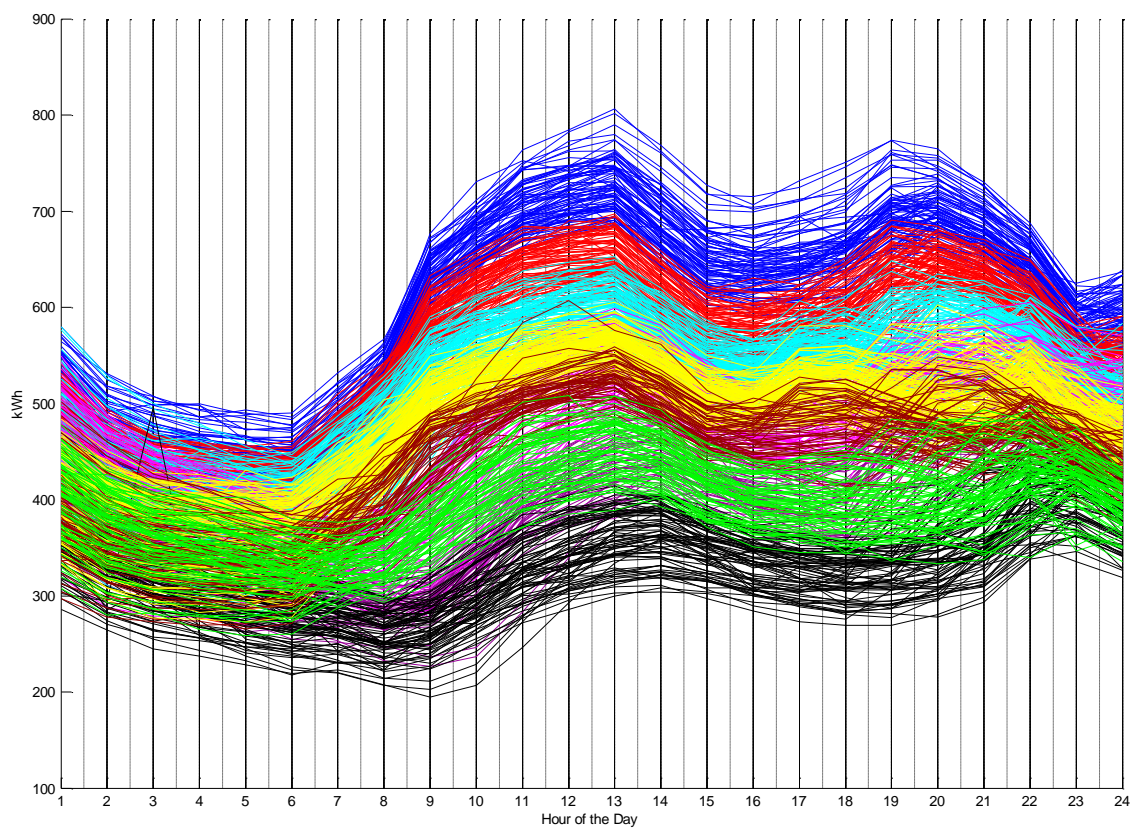

(b)

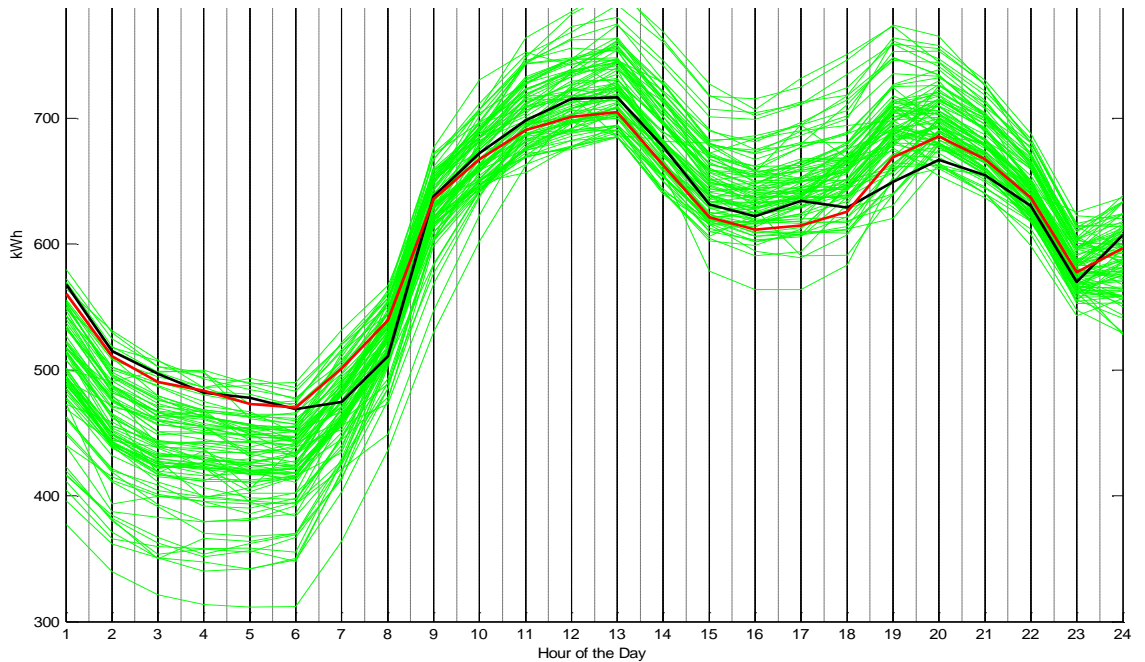

Figure 6. Load Forecasting results; (a) clustering of load curves ( 9 clusters, each of them represented by a different colour); (b) example of load forecasting for a day: target load in black, prediction in red, other load curves members of the cluster in green.

\section{Conclusion}

This paper has presented a MAS design to allow an easy implementation of VPPs. This distributed computing approach is capable of perfectly modelling the different entities involved in VPPs, facilitating their interaction and collaborative management. Applications of MAS have been reported in the literature, typically devoted to management of smart grid structures at large scales. In contrast, this work presents a detailed model for low-level management of VPPs. 
In order for VPP agents to be able to take informed choices, they need detailed information about what is happening in the network as well as predictions about what will potentially happen in the future. That is why this MAS design has been enriched with a demand forecasting algorithm based on Artificial Neural Networks that has been validated successfully with real data. Existing prediction algorithms have been utilized to facilitate predictions at a big, aggregated scale (nations, regions, etc.), but the one we propose forecasts load at a smaller, microgrid scale, that better fits the VPP size. Evaluation of the implementation results in errors around 1.5\%, which are low enough to feed the agents of the MAS with relevant data and allow them to make informed decisions.

\section{Acknowledgements}

We would like to express our thanks to the coordinators of the project OptimaGrid for the information provided on MAS-based micro-grids, and the creators of the MAS INGENIAS methodology.

This paper has been partially funded by the project SociAAL (Social Ambient Assisted Living), supported by Spanish Ministry for Economy and Competitiveness, with grant TIN2011-28335-C02-01, by the Programa de Creación y Consolidación de Grupos de Investigación UCM-Banco Santander for the group number 921354 (GRASIA group).

\section{References}

[1] Coster, E.J.; Myrzik, J.M.A.; Kruimer, B.; Kling, W.L.; Integration Issues of Distributed Generation in Distribution Grids. Proceedings of the IEEE, 99(1), 28-39. January 2011

[2] Rahimi, F.; Ipakchi, A.; Demand Response as a Market Resource Under the Smart Grid Paradigm. IEEE Transactions on Smart Grid 2010, 1(1), 82-88.

[3] Baladrón, C.; Aguiar, J.M.; Gobernado, J.; Carro, B.; Sánchez, A.; User-driven context aware creation and execution of home care applications. Annals of Telecommunications 2010, 65(9-10), $545-556$.

[4] Dae-Man Han; Jae-Hyun Lim; Design and implementation of smart home energy management systems based on zigbee. IEEE Transactions on Consumer Electronics 2010, 56(3), 1417-1425.

[5] Wille-Haussmann, B.; Erge, T.; Wittwer, C.; Decentralised optimisation of cogeneration in virtual power plants. Solar Energy 2010, 84(4), 604-611.

[6] McArthur, S.D.J.; Davidson, E.M.; Catterson, V.M.; Dimeas, A.L.; Hatziargyriou, N.D.; Ponci,

F.; Funabashi, T.; Multi-Agent Systems for Power Engineering Applications-Part I: Concepts, 
Approaches, and Technical Challenges. IEEE Transactions on Power Systems 2007 22(4), 17431752.

[7] Duan R. Multi-agent coordination in market environment for future electricity infrastructure based on microgrids. Conference: Systems, Man and Cybernetics, IEEE, 2009

[8] Pinto, T., Vale, Z.A, Morais, H., Praca, I., Ramos, C. : Multi-agent Based Electricity Market Simulator With VPP: Conceptual and Implementation Issues. Inc: 2009 IEEE PES General Meeting, 2009.

[9] Wang, Y.; Xia, Q.; Kang, C. Secondary Forecasting Based on Deviation Analysis for ShortTerm Load Forecasting. IEEE Trans. Power Systems 2011, 26(2), 500-507.

[10] P. McDaniel, S. McLaughlin. Security and Privacy Challenges in the Smart Grid. IEEE Security \& Privacy, 2009, 7(3), 75-77.

[11] H. F. Rashvand, K. Salah, J. M. Alcaraz Calero, L. Harn. Distributed security for multi-agent systems: review and applications. IET Information Security. IET. Dec. 2010, 4(4), 188-201.

[12] Jaime Lloret, Marc Gilg, Miguel Garcia, and Pascal Lorenz, A Group-based Protocol for Improving Energy Distribution in Smart Grids, IEEE International Conference on Communications (ICC 2011), Kyoto (Japan), June 5-9, 2011.

[13] Fernandez, C. R.; Gomez Sanz, J. J., Self-Management Capability Requirements with SelfMML \& INGENIAS to attain Self-Organising behaviours, Second International Workshop on Self-Organizing Architectures, 7th International Conference on Autonomic Computing and Communications, ICAC 2010, Washington DC, June 2010.

[14] Hernández, L.; Baladrón, C.; Aguiar, J.M.; Calavia, L.; Carro, B.; Sanchez-Esguevillas, A.; Cook, D.J.; Chinarro, D.; Gómez, J.; A Study of the Relationship between Weather Variables and Electric Power Demand inside a Smart Grid/Smart World Framework. Sensors 2012, 12(9), 11571-11591. 